\title{
Mechanosensitive $\beta$-catenin signaling regulates lymphatic vascular development
}

\author{
Boksik Cha ${ }^{1} \mathcal{E}$ R. Sathish Srinivasan ${ }^{1,2, *}$ \\ ${ }^{1}$ Cardiovascular Biology Research Program, Oklahoma Medical Research Foundation, ${ }^{2}$ Department of Cell Biology, University of Oklahoma \\ Health Sciences Center, Oklahoma City, Oklahoma 73104, USA
}

The Wnt/ $\beta$-catenin signaling is an evolutionarily conserved pathway that plays a pivotal role in embryonic development and adult homeostasis. However, we have limited information about the involvement of $W n t / \beta$-catenin signaling in the lymphatic vascular system that regulates fluid homeostasis by absorbing interstitial fluid and returning it to blood circulation. In this recent publication we report that canonical Wnt/ $\beta$-catenin signaling is highly active and critical for the formation of lymphovenus valves (LVVs) and lymphatic valves (LVs). $\beta$-catenin directly associates with the regulatory elements of the lymphedema-associated transcription factor, FOXC2 and activates its expression in an oscillatory shear stress (OSS)-dependent manner. The phenotype of $\beta$-catenin null embryos was rescued by FOXC2 overexpression. These results suggest that $\mathrm{Wnt} / \beta$-catenin signaling is a mechanotransducer that links fluid force with lymphatic vascular development. [BMB Reports 2016; 49(8): 403-404]

Wnt/B-catenin signaling is evolutionarily conserved among a variety of species and regulates diverse biological processes such as cell proliferation, apoptosis, cell polarity, cell differentiation, and migration $(\mathrm{H}$. Clevers \& R. Nusse, Cell, 2012 Jun 8;149(6):1192-205. doi: 10.1016). Not surprisingly, $\mathrm{Wnt} / \beta$-catenin signaling in one way or another influences the development of nearly every organ in the animal body. In

*Corresponding author. E-mail: Sathish-Srinivasan@omrf.org

http://dx.doi.org/10.5483/BMBRep.2016.49.8.112

\section{Received 14 July 2016}

Keywords: FOXC2, Lymphatic valves, Lymphovenous valves, Wnt/ $\beta$-catenin signaling

Abbreviations: ECs, endothelial cells; LVs, lymphatic valves; LVVs, lymphovenus valves; OSS, oscillatory shear stress

Perspective to: Cha et al (2016) Mechanotransduction activates canonical Wnt/ $\beta$-catenin signaling to promote lymphatic vascular patterning and the development of lymphatic and lymphovenous valves, Genes Dev. 2016 Jun 15;30(12):1454-69. doi: 10.1101/ gad.282400.116. Epub 2016 Jun 16. addition, aberrant $\mathrm{Wnt} / \beta$-catenin signaling is associated with a variety of diseases including cancer, osteoporosis, diabetes, and Alzheimer's disease.

Wnt/ $\beta$-catenin signaling is regulated at multiple levels (B.T. MacDonald et al., Dev Cell, 2009 Jul;17(1):9-26). Wnt ligands, receptors, co-receptors and mediators are regulated at both transcriptional and post-translational levels. In the absence of Wnt ligands, $\beta$-catenin, which is the centerpiece of the Wnt/ $\beta$-catenin signaling, remains as cytoplasmic and membrane bound. $\beta$-catenin associates with cadherins and is an integral part of the adherin junctions that mediate cell-cell and cell-matrix interaction. $\beta$-catenin translocates to the nucleus when Wnt ligands bind to the Frizzled/Lrp receptor/coreceptor complex. Inside the nucleus, $\beta$-catenin associates with the TCF/LEF family of transcription factors to activate their target genes.

Due to membrane localization of $\beta$-catenin, and its interaction with adherens junctions in association with critical role in $\mathrm{Wnt} / \beta$-catenin signaling, the protein has been long suggested as a mechanotransducer (B.M. Gumbiner, Cell, 1996 Feb 9;84(3):345-57). $\beta$-catenin might sense cell-extrinsic physical forces and translate them into cell-intrinsic signals. Our recent identification of a role played by $\beta$-catenin in lymphatic vascular morphogenesis might be strong evidence in favor of "mechanotransduction" model.

Lymphatic vascular system regulates fluid homeostasis by absorbing interstitial fluid and returning it back to blood circulation $(\mathrm{H}$. Chen et al, Microvasc Res, 2014 Nov;96:16-22. doi: 10.1016). Lymphatic vasculature is also critical for absorbing digested lipids from the intestine and for immune cell trafficking. The fluid within the lymphatic vessels is commonly known as the lymph. Genetic disorders or surgical and radiological damage to lymphatic vessels leads to lymphedema, a disease characterized by the swelling of tissues. Lymphedema patients are prone to infections and inflammations. There is currently no cure for lymphedema and only palliative treatments like massages are available. Understanding the mechanisms that regulate lymphatic vascular development might provide an opportunity to treat lymphedema.

Lymphatic endothelial cells (LECs) that originate predominantly from embryonic veins undergo stepwise morphogenesis to form the lymphatic vasculature. In the mature lymphatic 


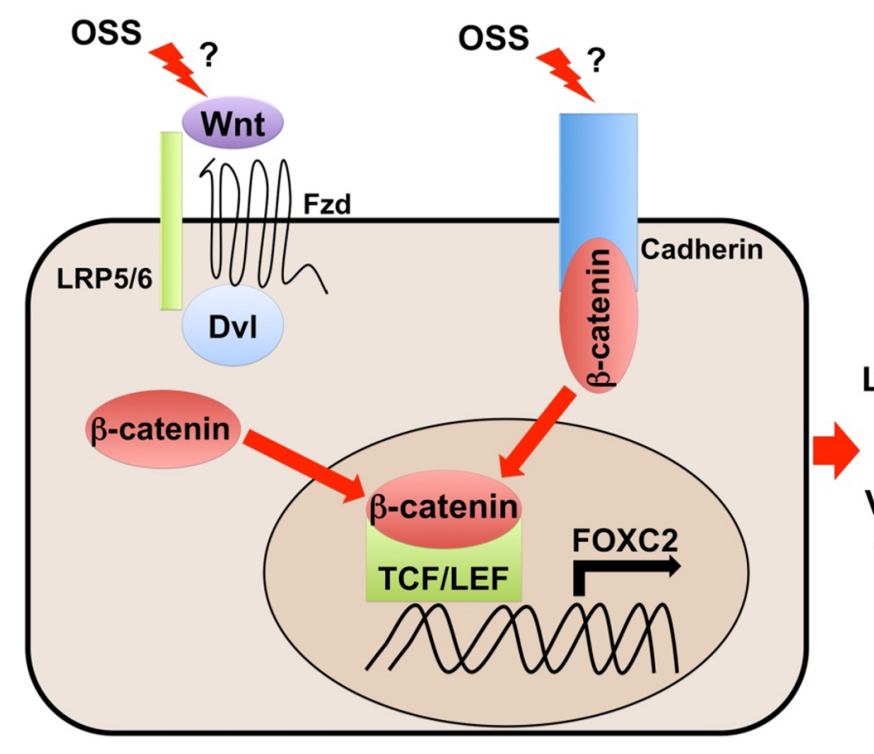

vasculature, lymphatic capillaries absorb interstitial fluid and drain it into the collecting lymphatic vessels. Lymphatic valves (LVs) within the collecting vessels regulate unidirectional flow of the fluid. Finally, lymph is returned into blood circulation at the junction of jugular and subclavian veins via two pairs of lymphovenus valves (LVVs). As LECs are constantly exposed to oscillatory lymph flow, lymphatic vascular development and homeostasis, they are speculated to be force regulated. Specifically, oscillatory shear stress (OSS) is known to promote the expression of lymphedema associated transcription factor, FOXC2. However, the manner in which OSS is sensed and translated into FOXC2 expression is still unknown.

In this study, we report that canonical $\mathrm{Wnt} / \beta$-catenin signaling is highly active and critical for LVVs and LVs formation. LEC-specific deletion of $\beta$-catenin in mouse embryos resulted in severe lymphedema. Further analysis revealed defective nature of lymphatic vessels and absence of LVVs and LVs. These phenotypes were reminiscent of Foxc2 $2^{-1-}$ embryos. Consistently, FOXC2 expression was defective in the LECs of mice lacking $\beta$-catenin. And, ectopic expression of FOXC2 partially yet dramatically rescued the lymphatic vascular phenotypes of mice lacking $\beta$-catenin. Furthermore, $\beta$-catenin directly associates with the regulatory elements of FOXC2 in LECs. Importantly, OSS activated the expression of $\beta$-catenin and FOXC2 in LECs. Inhibition of

\section{Lymphatic vascular patterning \\ $\&$}

\section{Valvular endothelial cell differentiation}

Fig. 1. A Model for the mechanosensitive $\beta$-catenin signaling during lymphatic vascular development. OSS may trigger $\beta$-catenin activation either in Wnt ligands dependent manner or in Wnt ligands independent manner.

Wnt/ $\beta$-catenin signaling with small molecules prevented the activation of FOXC2 expression by OSS. These results strongly suggest that $\mathrm{Wnt} / \beta$-catenin signaling is mechanically activated by fluid flow in LECs (Fig. 1).

Interesting questions still remain to be answered. We still do not know whether $\beta$-catenin directly senses fluid flow at LEC membrane or if additional intermediate sensors are involved. We cannot completely rule out the contribution of $\beta$-catenin in adherens junctions during lymphatic vascular morphogenesis. And, we also do not know if any Wnt-ligands are involved in this process. Further dissection of this powerful signaling pathway using genetic and molecular tools are necessitated to address these questions and test the "mechanotransduction" model. Importantly, we anticipate that numerous small molecule agonists and antagonists of $\mathrm{Wnt} / \beta$-catenin signaling might provide a prospect to treat lymphedema in the not so distant future.

\section{ACKNOWLEDGEMENTS}

RSS is supported by NIH/NHLBI (R01HL131652), institutional funds of OMRF, Oklahoma Center for Adult Stem Cell Research (OCASCR, 4340) and American Heart Association (15BGIA25710032). BC is supported by a post-doctoral fellowship from American Heart Association (15POST25080182). 